\title{
Regional Ecological-Economic Risk as a Criterion of Quality of Infrastructure of River Watercatchment Areas
}

\author{
Mavlyautdinova G.S.
}

Kazan Federal University, Institute of Management, Economics and Finance, Kazan, 420008, Russia

Email: gulnare_m@mail.ru

Pratchenko O.V.

Kazan Federal University, Institute of Language, 420008, Kazan, Russia

\section{Doi:10.5901/mjss.2015.v6n3p756}

\begin{abstract}
Introduction of the economic mechanism of environmental management at the meso-level, with the assessment of regional ecological-economic risks as the most important element of which, is one of the most important conditions of the successful implementation of the state natural resource policy. The regional ecological-economic risk is understood as generalized characteristic of different situations on this or that territory and during this period of time, the different degree of probability of transformation of the considered natural-economic system into a qualitatively different state is the result of which. The article presents new requirements to regional systems of environmental management.
\end{abstract}

Keywords: regional ecological-economic system, regional ecological-economic risk, water catchment area of the river, factor analysis, calculation of risks, risk assessment.

\section{Introduction}

In the current economic conditions there is great necessity for the development of scientifically based, fully verified methodology of the management development of Russia to ensure its stability and security of the whole country and its regions. In this connection there is the necessity to hold the research devoted to the problems of system diagnostics of regional ecological-economic systems, to form strategies of their sustainable and balanced development, the development of the appropriate methodology, mathematical algorithms and tools. Development of ecological-economic concept of water catchment areas as elementary topological units of the economic system, which are naturally subordinate in the geographical environment, if the part of this study. It is assumed that the risk calculation can be the measure (scale) for quantitative measurement of sustainable development of spatial ecological-economic systems, and the system of triggers connected with analysis and assessment of risks of special fuzzy logic, can serve as a management tool.

Algorithm and tools of the system diagnostics of complex systems, mathematical modeling and control of the dynamics of development of the regional ecological-economic systems based on synergetic theory and nonlinear dynamics, are presented in the works of P. Bergé, T. Buzan, K. Vidal, E. Peters, I. Pomo, R. Solso, I. Stengers, A. Frost, $\mathrm{H}$. Haken, and others. The statements of the theory of the agricultural standort J. Thünen, the rational standort of the industrial enterprise W. Launhardt, of the theory of the industrial standort A. Weber theory, the Central place theory of W. Christaller, the theory of regional markets and the spatial theory of prices by A. Cournot, the theory of absolute and comparative advantages of Adam Smith and David Ricardo, the theory of Heckscher-Ohlin, the doctrine of the spatial organization of the economy of A. Lösch and others $[1,2,3]$ made great contribution into the development of modern ideas concerning the laws and the regulations of functioning and development of the regional economy. The works of Russian scientists I.G. Aleksandrov, N.G. Bagautdinova, N.N. Baransky, P.I. Burak, I.R. Gafurov, V.S. Nemchinov, A.E. Probst, R.I. Shniper and others made a significant contribution to the development of ideas concerning the regional economy $[4,5,6,7,8,9]$. However, questions of the theory and practice of assessment and management of ecologicaleconomic risks within some watersheds, as well as with the account of the main infrastructural peculiarities of different water-catchment areas, questions of allocation of representative integral indicators that are able to characterize the current ecological-economic condition of water catchment areas, its spatial and temporal dynamics were understudied. 
This caused the necessity for further studies.

\section{Methodology}

Task management regional ecological-economic risks (EAR) includes three successive stages: 1) multidimensional assessment of events and phenomena of the system with a representative selection of variables; 2) determination of the probabilistic risk of destabilizing the system state in the form of quantitative values; 3) analysis of the result and its interpretation in the form of a qualitative assessment.

As part of the study, analysis and integration of long-term series (2001-2010) of the ecological-economic information that reflects the functioning and condition of different aspects of ecological-economic infrastructure of watercatchment areas of 49 small rivers of the Republic of Tatarstan, were carried out. Since most of the parameters under consideration is are connected to the administrative regions of the Republic, their values adjusted to the share of the territory of certain watersheds, were used to estimate the ecological-economic infrastructure. Water-catchments areas of the rivers were divided into lots located within a particular administrative district, with further calculation of the share of the lot the region area. This proportional coefficient corrected the absolute figures related to the administrative region as a whole. All figures are obtained from official sources: reference data and library materials, different forms of State statistical reporting.

\section{Results}

At the first stage of work the factor analysis of 161 indicators selected for the study was carried out. 3 factors were selected by the principal component method. Some factors were chosen by the "scree debris" method. The fact that some factors are orthogonal allowed to group on their basis three independent blocks of variables, conventionally called "impact on the system", "system productivity" and "system response".

Factor loadings were calculated for each variable participated in the analysis, and in this case the variables with factor loadings $\geq 0,7$, were selected in every factor. Factor loadings calculated as a result of the factor analysis are the correlation coefficients between the specific variables and factors, reflecting the contribution of every variable in their complex. This fact allows us to interpret factor loadings as the relative weighting coefficients of these variables. The ranged series of factor loadings was formed in order to convert them into weight coefficients, and values of factor loadings were "weighted" with respect to their maximum value: $\mathrm{K}_{\mathrm{fn}}=\mathbf{f}_{\mathrm{n}} / \mathrm{f}_{\mathrm{n}}$ max.

Values of representative variables selected by the factor analysis were also reduced to 1 :

Value_red $=$ Value $/$ Value_max,

and reduced values were multiplied by corresponding weight coefficients:

Indicator $=$ Value _red ${ }^{*} K_{\text {fn }}$.

The obtained data sets are normalized and weighted values of indicators of the state of the ecological-economic system of the water-catchment area. In this case, the calculated indicators had the normal trend which, if necessary, was provided by the inverting ( 1 - index), which allowed to obtain the single vector of orientation. For example, such indicators as "Woodiness" and "Erosional feature of lands" are oppositely oriented. To provide a single vector pointing at deterioration of condition with increasing values of the index, "Woodiness" should be presented as (1 - indicator of "Woodiness"). Inverted indicators had negative factor loadings.

For the analysis of any complex system it is necessary to reduce the indicators of functioning of different subsystems to a single scale, to determine the level of their contingency, interdependence, to "adjust" them to each other. Correlations of indicators of different subsystems with each other, significant regression models, multidimensional scaling and different variants of dispersion analysis of variance can serve as elements of setting. The task is that all these "adjusted" and scaled indicators should represent a single complex of environmental, economic and social factors.

Accounting and forecasting of probable transition of the system into non-equilibrium (equilibrium) state, in fact calculation and assessment of risks, is the best approach to achieve this goal. As far as the values of each indicator reduced to 1 are a specific value which reflects the share of these values from maximum, the comparison of the arithmetic average and the median of the whole series of different indicators obtained for the particular water-catchment area, allows to determine the shift of distribution of values downward or upward, their frequency asymmetry, being the probabilistic characteristic of the series.

The median (quantile 0,5 ) is a possible index value; it divides the range series (variation sample series) into two equal parts: $50 \%$ of all values of the variation series will be not more than the median, and $50 \%$ - not less than the median. The median is an important characteristic of distribution of the random variable and - as well as the 
mathematical expectation - can be used in centering of distribution.

Median is more robust, that is less sensitive to different deviations and inhomogeneity in the sample than the arithmetic average or expectation value and, therefore, is more preferable to assess the distribution centers. Besides, in case of uncertainty, the median is redefined, while the expectation value cannot be determined (for example, the Cauchy distribution) [10].

The method of calculation of the first quartile (25\%) in the ranked series of the same type of sample of calculated medians was used to find the background level of indicators that will serve as a characteristic of the upper limit of acceptable risk. The index value taken as a background one is the basis on which the risk is calculated. Dimensionless values that were reduced to corresponding probability values expressed in fractions using the nonlinear function [6], are obtained in the result of division of values calculated for specific watersheds, on the background ones:

Riski=1- $\exp \left(-\left(\left(m_{i} / m_{\phi}\right)^{2} / 2\right)\right)$,

where

Risk $\mathbf{k}_{\mathbf{i}}$ - statistical probability of exceeding of a specific risk indicator above the background value in the i-segment (probabilistic risk);

$\mathrm{m}_{\phi}-$ background indicator value corresponding to the first quartile;

$m_{i}-$ value of the indicator in the i-segment.

3 indicators of risk were obtained as a result of all transformations: 1) risk of overexposure on the system (Riskesp); 2) risk of insufficient stability of the system to the exposure (Riskinst); 3 ) risk of production loss of the system (Riskinef).

The ratio of all the risks was transformed into the generalized EER with the help of the power function of the form $\left(\mathrm{R}_{1}{ }^{*} \mathrm{R}_{2}{ }^{*} \ldots{ }^{*} \mathrm{R}_{n}\right)^{1 / n}$ :

ERR $=\left(\text { Risk }_{\text {exp }}{ }^{*} \text { Riskinst }^{*}{ }^{*} \text { isk } \text { inef }\right)^{1 / 3}$

Correlation of calculated risks is shown on Figure 1 on which the curves of risk values are presented as polynomials.

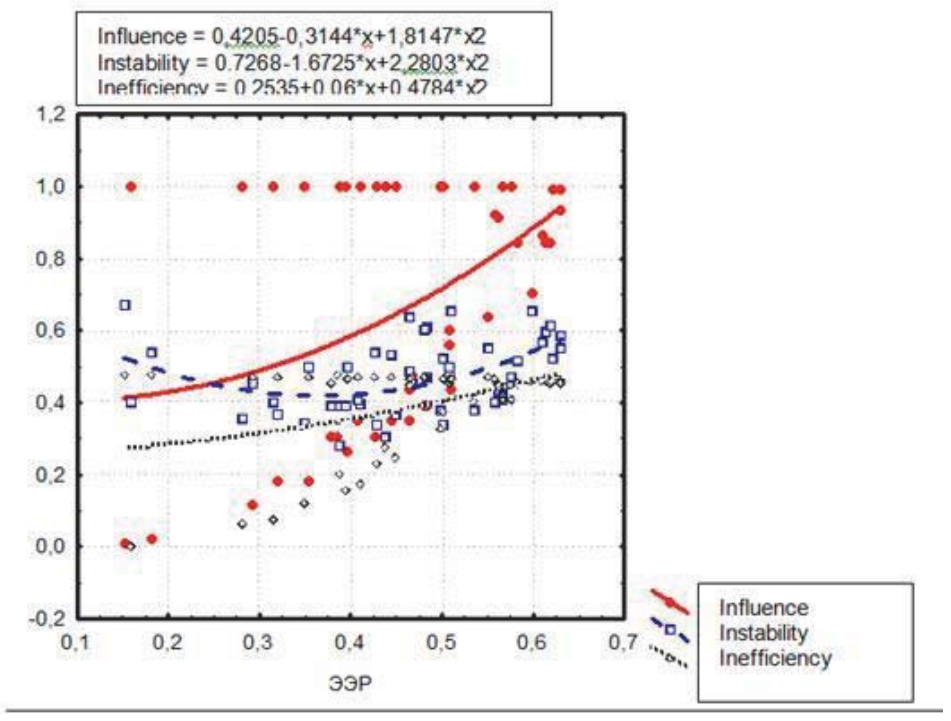

Fig.1. Correlation and balance of risks presented in the form of polynomials

EER values were divided into 4 ranks corresponding to 4 gradations of risk - low, medium, high and critical - by the Method of quartiles (25\%, $50 \%, 75 \%$ percentiles).

ERR Gradation: Low risk EER <0,387; Medium risk 0,387 $\leq$ EER $<0,481$; High risk $0,481 \leq$ EER $<0,565$; Critical risk $0,565 \leq E E R$. The higher the values of ERR calculated for the particular water-catchment area, the more strained the situation is and the higher the risk of the system destabilization is.

High values of risk indicate the risk of exposure to excessive loads experienced by the ecological-economic system. High risk of instability indicates the decrease of the system resistance. High risk of inefficiency indicates the loss of production and economic efficiency of water-catchment infrastructure [11].

Since on the considered watersheds the economy is represented mainly by agriculture, exactly the indicators 
characterizing the agricultural development of the territory were considered. Analysis of various regressions with stepwise inclusion of variables into the models that assessed their contribution into the value of such parameter as "Operating income of agriculture", allowed to set up the equation of multiple regression, which is statistically significant and which comprehensively covers the observed variability:

Agricultural profit (thousand rubles) $=1165,3^{*}$ Potato yielding capacity (c/ha) $-7,0 *$ Bird cost value (rubles) 1849,1*Polluted water removal $\left(\mathrm{mln} \cdot \mathrm{m}^{3}\right)-123534,5$.

The model parameters are the following: the multiple correlation coefficient $R=0,73$; determination coefficient (that characterizes the coverage ratio of "explained" dispersion expressed in fractions 1) $R^{2}=0,53$; criterion $F=38,3$; level of significance $p=0,008$. It is important to note that the regression coefficient of polluted waters has a negative value, reducing the total score in the equation.

Multiple regression equation set up for such indicator as "Profitability of agriculture" was another interesting model:

Cost effectiveness of agriculture (\%) $=69,7-0,0034^{\star}$ Cattle performance cost value (rub.) $-0,09 \star$ Supply of land (thousand ha) $-0,24^{*}$ Cost of production of 1 beetroot hundredweight (rub.) $+1,116^{*}$ Spent water $\left(\mathrm{mln} \cdot \mathrm{m}^{3}\right)+0,0004^{*}$ Stock of cattle (animal units) $-0,0002^{*}$ Stock of pigs (animal units) $-0,0087^{*}$ Costs for environmental protection (mln.rub.) $-1,07^{\star}$ water withdrawal (mln. $\left.\mathrm{m}^{3}\right)-0,0027^{*}$ Cost price of eggs (rub.).

Characteristics of the model: $R=0,75 ; R^{2}=0,56 ; F=14,7 ; p=0,0004$.

It is necessary to note that withdrawal and use of water in the equation are evaluated in the opposite way: the high level of water withdrawal itself reduces the profitability of production, but involvement in the production and increase in volume of water use proportionally increases profitability. Regression coefficients are $-1,070$ and $+1,116$, respectively.

In the analysis of the correlation matrix we pay attention to the fact that the risk of exposure is accompanied to a certain extent by increased productivity of the system, as is evidenced by the inverse correlation with the risk of inefficiency $(r=-0,56, p=0,00012)$. Likewise, the impact on the system stimulates its response, reducing the risk of instability: $r=-0,48, p=002$. Therefore, in the result exactly the risk of exposure mainly determines the generalized EER $(r=0,78, p=0,0001)$.

On the other hand, the profit is connected with all calculated risks in the following way:

Cost effectiveness of agriculture (thousand rub.) $=163683,7+9823,4 *$ Riskexp $-85824,8 *$ Riskinst $255197,7^{*}$ Riskinef; $\left(R=0,77 ; R^{2}=0,59 ; F=42,7 ; p<0,001\right)$.

Taking into account that values of all risks are reduced to the single scale, their regression coefficients are a measure of contribution of every risk into the final result. As we can see from the equation, the risk of inefficiency makes the greatest contribution to the variability of profit, then - the risk of instability and only then - the risk of exposure. That is - although the impact on the system itself provides the profit growth - but it is not a determining factor; indicators that affect the stability and effectiveness of ecological-economic system are more important. In other words, the impact on the system makes a profit only in case of sufficient stability, and this condition is reflected in the effectiveness of the system.

\section{Conclusion}

Thus, the assessment of relative risk as a variety of probabilistic risk, considers the relationship between objects under the influence and without it. The value of relative probability risk, to which the ecological-economic risk can be referred, makes it possible to determine how many times the probability of display of signs negative for the system, caused by the certain influence, increases.

Fig.1 Influence, Instability, Inefficiency

\section{References}

Bagautdinova N.G., Gafurov I.R., Novenkova A.Z. The transformation of region's economic area governed by the development of industrial region // World Applied Sciences Journal, 25(7), 2013, 1113-1117.

Bagautdinova, N.G., Panasyuk, M.V., Gafurov, I.R. Wavelet analysis of the territorial socio-economic system dynamics // World Applied Sciences Journal, 27(13), 2013, 62-66.

Bagautdinova, N., Gafurov, I., Kalenskaya, N., Novenkova, A. The regional development strategy based on territorial marketing (The Case of Russia) // World Applied Sciences Journal, 18(Issue SPL.ISSUE. 18), 2012, 179-184Krugman P. Space: the last frontier. // Spatial economy, 2005. - №3. - pp. 121-126.

Lesh A. Geographical placement of economy: Translation from English. - M.: Publishing house of foreign literature, 1959.

Glebova I.S., Khabibrakhmanova R. and Yasnitskaya Y. The Analysis of the Impact of the Investment Attractiveness Factors of the Region on the Fixed Capital Investments in the Economy of the Republic of Tatarstan II Middle-East Journal of Scientific 
Research 17 (10): 1498-1502, 2013.

Barro R., Sala-i-Martin X Economic Growth. 2nd ed. The MIT Press, 2004.

Borts G.H., Stein J.I. Economic Growth in a Free Market. - New York, London, 1964.

Fujita M., Krugman P. When is the economy monocentric: von Thunen and Chambertin unified // Regional Science and Urban Economics, 1995.

Robert G. Staudte: Robust estimation and testing. Wiley, New York 1990.

Fredann J. Urbaniezention Planing and Naitional Development. - Beverly Hills, London, 1973.

Kirshin I.A., Datsyk A.A., Titov A.V. Forecasting the Dynamics of an Innovative Cycle. - World Applied Sciences Journal (Economics, Management and Finance). - 2013. - №27. - P. 197 - 201.

Ricardo D. Beginning of political economy and tax load // Writing. V. I., M.: State publishing house of political literature, 1955. $-360 \mathrm{p}$.

Smith A. Investigation on nature and reasons of nation riches. - M.: Publishing house of social-economic literature, $1962,-684 \mathrm{p}$.

Glebova I.S., Sadyrtdinov R. and Rodnyansky D. Impact Analysis of Investment Attractiveness of the Republic of Tatarstan on Fixed Investments of its Leading Companies // World Applied Sciences Journal 26 (7): 911-916, 2013. 